\title{
Safe chest tube placement in a patient with tension pneumothorax receiving rivaroxaban therapy for non-valvular atrial fibrillation
}

\author{
Alessandro Cipriano, Matteo Parollo, Ester Taurino, Elena Venturi, Massimo Santini \\ Emergency Department, Azienda Ospedaliero-Universitaria Pisana, Pisa University Hospital, Italy
}

\begin{abstract}
The number of patients treated with direct oral anticoagulants is increasing worldwide. Although bleeding complications associated with direct oral anticoagulants are lower than those associated with vitamin $\mathrm{K}$ antagonists, the increased number of patients treated with these anticoagulants suggests that a higher absolute number of patients are at risk. Tube thoracostomy is an invasive procedure with a high risk of bleeding. To date, among direct oral anticoagulants, only dabigatran has a well-studied antidote to reverse its effects during emergency procedure or surgery. This report describes a case in which emergency placement of a tube thoracostomy, in a patient with type 2 respiratory failure due to
\end{abstract}

Correspondence: Alessandro Cipriano, Emergency Department, Azienda Ospedaliero-Universitaria Pisana, Pisa University Hospital, Via di Piaggetta Traversa A 28, 56121 Pisa, Italy.

Tel. +39.3471758192 - Fax: +39.050.997351.

E-mail: a.cipriano@ao-pisa.toscana.it

Key words: Pneumothorax; rivaroxaban; thoracostomy; anticoagulants; bleeding.

Authors' contributions: AC, MP, ET, EV, were present during the medical case and drafted the manuscript; AC, MP, analyzed and wrote the literature review; $\mathrm{AC}, \mathrm{MS}$, contributed to manuscript revision and take responsibility for the paper as a whole.

Conflict of interest: AC and MS received grants as consultant to these pharmaceutical industries: Boehringer Ingelheim, Pfizer, Bayer, Aspen, Daiichi Sankyo. The other authors have no conflict of interest to declare.

Funding: This research did not receive any specific grant from funding agencies in the public, commercial, or not-for-profit sectors.

Informed consent: the patient provided informed consent for publication of the case.

Received for publication: 1 March 2020

Accepted for publication: 4 May 2020.

${ }^{\circ}$ Copyright: the Author(s), 2020

Licensee PAGEPress, Italy

Monaldi Archives for Chest Disease 2020; 90:1262

doi: 10.4081/monaldi.2020.1262

This article is distributed under the terms of the Creative Commons Attribution Noncommercial License (by-nc 4.0) which permits any noncommercial use, distribution, and reproduction in any medium, provided the original author(s) and source are credited. left tension pneumothorax and receiving the anticoagulant rivaroxaban, in the pharmacokinetics phase with greater anticoagulant effect, did not result in bleeding greater than that typically encountered during such interventions. The procedure ended successfully with no acute complications.

\section{Introduction}

Direct oral anticoagulants (DOACs) use is increasing worldwide. Indications for DOAC treatment are diverse and consist of different medical conditions such as prevention of stroke and systemic embolism in non-valvular atrial fibrillation or treatment and secondary prevention of venous thromboembolism [1]. Currently licensed DOACs include the factor Xa inhibitors, apixaban, rivaroxaban and edoxaban, and the thrombin inhibitor, dabigatran, and have largely replaced other anticoagulants such as vitamin $\mathrm{K}$ antagonists (VKAs). Advantages of DOACs compared to VKAs are the predictable pharmacokinetics with simplified dosing, the lower interactions with other drugs or food, and that routine monitoring by coagulation tests is not necessary [2].

The number of patients treated with direct oral anticoagulants (DOACs) is increasing worldwide [1-3]. Recent data suggest that over 2.9 million US patients take one of the factor Xa inhibitors [3]. Although bleeding complications associated with DOACs are lower than those associated with vitamin $\mathrm{K}$ antagonists [4,5], the increased number of patients treated with anticoagulants suggests that a higher absolute number of patients are at risk. Importantly, anticoagulant drugs have been responsible for more adverse drug reaction visits to emergency departments than any other class of medication [6].

Although routine coagulation tests are available, they are inadequate for optimal care. DOAC testing may be useful in the context of critical clinical situations, such as life-threatening hemorrhage or conditions that require emergency surgery. DOAC-specific tests have been developed but have limited availability in both Europe and the United States [7]. Therefore, if minor or major elective surgery is imminent, there might be uncertainties regarding the optimal perioperative management of patients receiving DOACs. Moreover, to date, only dabigatran, among direct oral anticoagulants, has a well-studied antidote to reverse its effects during emergency procedure or surgery $[8,9]$.

Pneumothorax comprises air in the intrapleural space and can range from benign to life-threatening severity. Pneumothorax can arise both as a primary spontaneous manifestation and as a consequence to a pre-existing lung disease [10]. COPD (chronic obstructive pulmonary disease) is the leading cause of secondary pneumothorax, patients with severe COPD carry the highest risk [10]. Tension pneumothorax is a life-threatening complication 
resulting from pressure in the pleural cavity causing hemodynamic compromise. This condition may require emergency treatment with tube thoracostomy, a procedure with a high risk of bleeding according to recent international guidelines [11], nevertheless rate of bleeding complication is not well evaluated and seems rare $(<1 \%)[12,13]$. In any case, primary or iatrogenic coagulopathy is an absolute contraindication to elective procedure and a relative contraindication to urgent one [11].

\section{Case Report}

A 63-year-old man was admitted to our second-level emergency department due to acute onset of dyspnea and thoracic oppression. His recent medical history included coughing and fever for 3 days prior to admission, as well as emergency department admission 4 months before the current episode for acute onset of chest pain, which was not diagnosed as acute or inducible heart ischemia or any other important urgent disease. His past medical history included acute myocardial infarction treated with percutaneous coronary intervention, hypertension, chronic ischemic heart disease, chronic obstructive pulmonary disease, paroxysmal atrial fibrillation, and spontaneous pneumothorax 20 years prior, which had been treated by drainage. His CHA2DS2VASc risk score was 2 (hypertension and vascular disease) and his HAS-BLED risk score was 1 (hypertension). He was taking the following medications: rivaroxaban $20 \mathrm{mg}$ (last dose $6 \mathrm{~h}$ prior to admission), metoprolol, salmeterol + fluticasone via inhaler, tiotropium, amiodarone, ramipril, and atorvastatin. First responders administered furosemide $80 \mathrm{mg}$ intravenously. On physical examination, he was alert, without apparent neurological deficits, and was standing in a semi-orthopneic position. Markedly reduced left basal lung sounds were observed and ventilation was severely impaired. His vital signs were: arterial pressure $220 / 120 \mathrm{mmHg}$, heart rate 110 beats/min, $\mathrm{SpO}_{2} 80 \%$, respiratory rate 32 breaths $/ \mathrm{min}$, and temperature $36.3^{\circ} \mathrm{C}$. Arterial blood gases and other blood parameters were as follows: $\mathrm{pH} 7.29, \mathrm{pCO}_{2} 64 \mathrm{mmHg}$, $\mathrm{pO}_{2} 36 \mathrm{mmHg}$, serum lactate $1.7 \mathrm{mEq} / \mathrm{l}$, bicarbonate $25.4 \mathrm{mmol} / \mathrm{l}$,

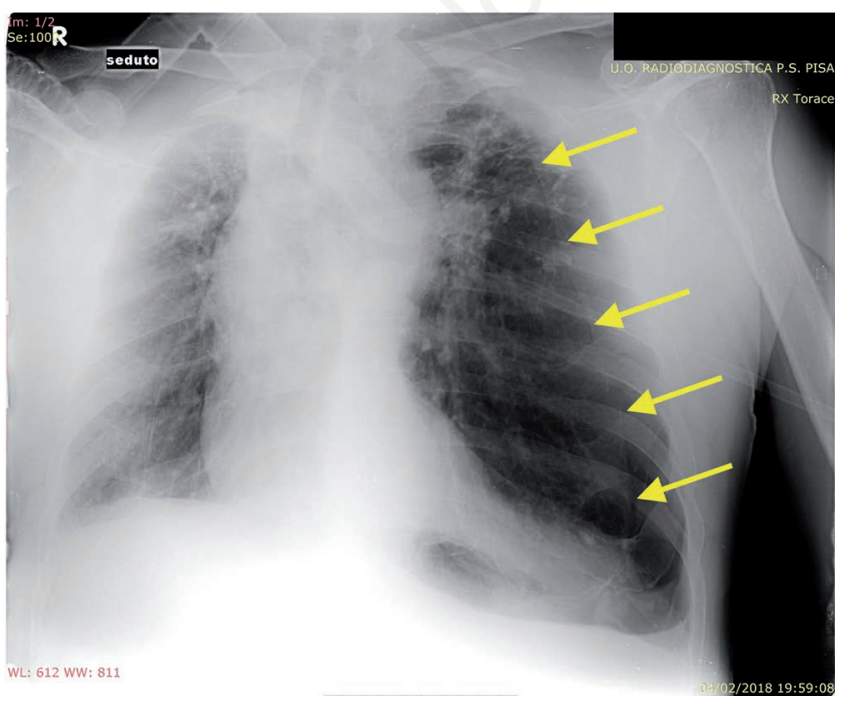

Figure 1. Chest X-ray at diagnosis.
$\mathrm{O}_{2}$ saturation $71.9 \%$, hemoglobin $17.2 \mathrm{~g} / \mathrm{dl}$, and glucose $115 \mathrm{mg} / \mathrm{dl}$. On electrocardiography, a sinus rhythm was detected. Salbutamol, methylprednisolone, ipratropium bromide, and oxygen via nonmechanical ventilation were administered. An urgent chest X-ray was performed, which detected left basal pneumothorax with concurrent contralateral heart deviation (Figure 1). The diagnosis was type 2 respiratory failure due to left tension pneumothorax.

The selected treatment was placement of a tube thoracostomy because we considered other solutions, like non-invasive-ventilation not useful or detrimental. Local and systemic analgesia were administered before and during the procedure: intravenous morphine and topical carbocaine. A horizontal 4-cm skin incision was made upon the rib delimiting the inferior margin of the 5th space. A 20-Fr tube was then inserted, advanced, and connected to drainage tubing and a drainage device.

The procedure was performed $6 \mathrm{~h}$ and $30 \mathrm{~min}$ after the last dose of rivaroxaban and ended successfully without acute complications or excessive bleeding; the patient experienced immediate resolution of dyspnea and normalization of vital signs. Arterial blood gases and other blood parameters were as follows: $\mathrm{FiO}_{2}$ $30 \%, \mathrm{pH} 7.35, \mathrm{pCO}_{2} 47 \mathrm{mmHg}, \mathrm{pO}_{2} 117 \mathrm{mmHg}$, serum lactate 1.9 $\mathrm{mEq} / 1, \mathrm{O}_{2}$ saturation $98.7 \%$, bicarbonate $24.5 \mathrm{mmol} / 1$, and hemoglobin $16.6 \mathrm{~g} / \mathrm{dl}$. X-ray confirmed correct positioning of the device (Figure 2). Laboratory tests showed prothrombin activity, $46 \%$; international normalized ratio, 1.69; and activated partial thromboplastin time, $41.8 \mathrm{~s}$.

The patient was transferred to the Thoracic Surgery Unit for monitoring and follow-up. He was treated with prophylactic dose of enoxaparin (40 mg day) till the chest tube was removed on day 5 (after complete pneumothorax resolution) and then shifted to dabigatran $150 \mathrm{mg}$ bid considering the risk of recurrence and the possibility to use an antidote. No further complications arose.

\section{Discussion}

Tube thoracostomy is an invasive procedure with a high risk of bleeding [11]. Emergency placement of a tube thoracostomy in this

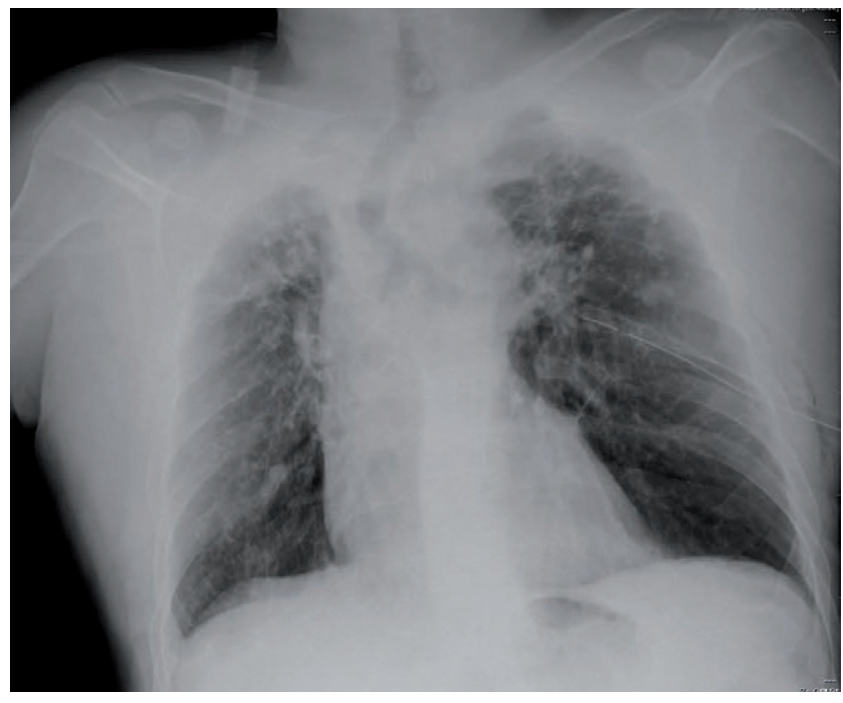

Figure 2. Chest X-ray after tube thoracostomy. 
patient treated with rivaroxaban $20 \mathrm{mg}$ (in the pharmacokinetics phase with greater anticoagulant effect) did not result in bleeding greater than that typically encountered during such interventions. This report emphasizes the superiority of DOACs compared to vitamin $\mathrm{K}$ antagonists, with respect to safety and ease of use. The decision to quickly use tube thoracostomy instead of an over-theneedle catheter was related to the well know high risk of failure. Due to the variable thickness of the chest wall, kinking of the catheter, and other technical or anatomic complications, needle decompression may not be successful [14]. Moreover, this patient was in a life-threatening condition that required no time-consuming procedures but a quickly and safe resolution. For these reasons we also decided not to use reversal agents, considering their not well proven efficacy in this setting [11].

To the best of our knowledge, no prior case reports have described tube thoracostomy placement in DOAC-treated patients with tension pneumothorax. Within the implicit limitations of a case report, our description of this case could help emergency physicians to have greater confidence in comparable settings.

\section{Conclusions}

Tension pneumothorax is a common life-threatening condition that promptly needs to be treated with chest tube placement, a high bleeding risk procedure for which experience in anticoagulated patients is lacking. This case report shows that this mandatory procedure was safe and feasible in a patient treated with rivaroxaban, without pre-treatment with currently available reversal agents.

\section{References}

1. Loo SY, Dell'Aniello S, Huiart L, Renoux C. Trends in the prescription of novel oral anticoagulants in UK primary care. Br J Clin Pharmacol 2017;83:2096-106.

2. Halvorsen S, Ghanima W, Tvete IF, et al. A nationwide registry study to compare bleeding rates in patients with atrial fibrilla- tion being prescribed oral anticoagulants. Eur Heart J Cardiovasc Pharmacother 2017;3:28-36.

3. Deitelzweig S, Neuman WR, Lingohr-Smith $M$, et al. Incremental economic burden associated with major bleeding among atrial fibrillation patients treated with factor Xa inhibitors. J Med Econ 2017;20:1217-23.

4. Ruff CT, Giugliano RP, Braunwald E, et al. Comparison of the efficacy and safety of new oral anticoagulants with warfarin in patients with atrial fibrillation: A meta-analysis of randomised trials. Lancet 2014;383:955-62.

5. Chai-Adisaksopha C, Hillis C, Isayama T, et al. Mortality outcomes in patients receiving direct oral anticoagulants: a systematic review and meta-analysis of randomized controlled trials. J Thromb Haemost 2015;13:2012-20.

6. Shehab N, Lovegrove MC, Geller AI, et al. US emergency department visits for outpatient adverse drug events, 20132014. JAMA 2016;316:2115-25.

7. Connors JM. Testing and monitoring direct oral anticoagulants. Blood 2018;132:2009-15.

8. Pollack CV Jr, Reilly PA, Van Ryn J ,et al. Idarucizumab for dabigatran reversal - Full cohort analysis. N Engl J Med 2017;377:431-41.

9. Connolly SJ, Crowther M, Eikelboom JW, et al. Full study report of andexanet alfa for bleeding associated with factor $\mathrm{Xa}$ inhibitors. N Engl J Med 2019;380:1326-35.

10. Marx J, Hockerberger R WR. Rosen's emergency medicine Concepts and clinical practice. 9th ed. Philadelphia, PA: Saunders; 2018: p. 881.

11. Steffel J, Verhamme P, Potpara TS, et al. The 2018 European Heart Rhythm Association practical guide on the use of nonVitamin $\mathrm{K}$ antagonist oral anticoagulants in patients with atrial fibrillation: executive summary. Europace 2018;20:1231-42.

12. Yacovone ML, Kartan R, Bautista M. Intercostal artery laceration following thoracentesis. Respir Care 2010;55:1495-98.

13. Wolfe KS and Kress JP. Risk of procedural hemorrhage. Chest 2016;150:237-46.

14. Stewart RM, Henry S, Brasel K. Advanced Trauma Life Support ${ }^{\circledR}$. 10th ed. Chicago: American College of Surgeons; 2018: p. 66. 